\title{
Extinction in Two-Species Nonlinear Discrete Competitive System
}

\author{
Liqiong Pu, ${ }^{1}$ Xiangdong Xie, ${ }^{2}$ Fengde Chen, ${ }^{2}$ and Zhanshuai Miao ${ }^{1}$ \\ ${ }^{1}$ College of Mathematics and Computer Science, Fuzhou University, Fuzhou, Fujian 350108, China \\ ${ }^{2}$ Department of Mathematics, Ningde Normal University, Ningde, Fujian 352300, China \\ Correspondence should be addressed to Xiangdong Xie; ndsyxxd@163.com
}

Received 13 December 2015; Revised 24 March 2016; Accepted 27 March 2016

Academic Editor: Cengiz Çinar

Copyright (c) 2016 Liqiong Pu et al. This is an open access article distributed under the Creative Commons Attribution License, which permits unrestricted use, distribution, and reproduction in any medium, provided the original work is properly cited.

\begin{abstract}
We propose a nonlinear discrete system of two species with the effect of toxic substances. By constructing a suitable Lyapunovtype function, we obtain the sufficient conditions which guarantee that one of the components will be driven to extinction while the other will be globally attractive with any positive solution of a discrete equation. Two examples together with their numerical simulations illustrate the feasibility of our main results. The results not only improve but also complement some known results.
\end{abstract}

\section{Introduction}

Let $Z$ denote the set of all nonnegative integers. For any bounded sequence $\{f(n)\}$, set $f^{u}=\sup _{n \in Z} f(n)$ and $f^{l}=$ $\inf _{n \in Z} f(n)$.

In the real world, there are many types of interactions between two species. Competitive relations are among the most common ecological interactions. As we all know, the competitive system has been established and was accepted by many scientists and now it became the most important means to explain the ecological phenomenon. During the last decade, the study of the dynamic behaviors of competitive system with toxic substance or feedback control have been discussed by many authors; see, for example, [1-16]. However, most of the studies are based on the traditional Lotka-Volterra competitive system $[5,7,8,17-20]$; seldom did scholars consider the nonlinear case $[1-4,8,9,11,12,15,16,21-25]$.

In [1], Li and Chen studied the extinction property of the following two species competitive system:

$$
\begin{aligned}
& \dot{x}_{1}(t)=x_{1}(t)\left[r_{1}(t)-a_{1}(t) x_{1}(t)-b_{1}(t) x_{2}(t)\right. \\
& \left.\quad-c_{1}(t) x_{1}(t) x_{2}(t)\right], \\
& \dot{x}_{2}(t)=x_{2}(t)\left[r_{2}(t)-a_{2}(t) x_{1}(t)-b_{2}(t) x_{2}(t)\right. \\
& \left.\quad-c_{2}(t) x_{1}(t) x_{2}(t)\right],
\end{aligned}
$$

where $r_{i}(t), a_{i}(t), b_{i}(t), c_{i}(t), i=1,2$, are assumed to be continuous and bounded above and below by positive constants and $x_{1}(t), x_{2}(t)$ are population density of species $x_{1}$ and $x_{2}$ at time $t$, respectively.

In fact, when the size of the population is relatively small, the discrete time models governed by difference equations are more appropriate than the continuous ones. Therefore, $\mathrm{Li}$ and Chen [2] and Guo et al. [3] studied the following discrete Lotka-Volterra competition system:

$$
\begin{aligned}
& x_{1}(n+1)=x_{1}(n) \exp \left[r_{1}(n)-a_{1}(n) x_{1}(n)\right. \\
& \left.\quad-b_{1}(n) x_{2}(n)-c_{1}(n) x_{1}(n) x_{2}(n)\right], \\
& x_{2}(n+1)=x_{2}(n) \exp \left[r_{2}(n)-a_{2}(n) x_{1}(n)\right. \\
& \left.\quad-b_{2}(n) x_{2}(n)-c_{2}(n) x_{1}(n) x_{2}(n)\right],
\end{aligned}
$$

where $\left\{r_{i}(n)\right\},\left\{a_{i}(n)\right\},\left\{b_{i}(n)\right\}$, and $\left\{c_{i}(n)\right\}, i=1,2$, are bounded nonnegative sequences defined on $n \in Z$. In [2], Li and Chen showed that if the coefficients of system (2) satisfy

$$
\frac{r_{2}^{u}}{r_{1}^{l}}<\min \left\{\frac{a_{2}^{l}}{a_{1}^{u}}, \frac{b_{2}^{l}}{b_{1}^{u}}, \frac{c_{2}^{l}}{c_{1}^{u}}\right\},
$$


species $x_{2}$ will be driven to extinction. In [3], Guo et al. introduced the average growth rate and showed that if the coefficients of system (2) satisfy the following inequality:

$$
\frac{M\left[r_{2}\right]}{m\left[r_{1}\right]}<\min \left\{\frac{a_{2}^{l}}{a_{1}^{u}}, \frac{b_{2}^{l}}{b_{1}^{u}}, \frac{c_{2}^{l}}{c_{1}^{u}}\right\},
$$

then the same conclusion holds. Obviously, condition $\left(H_{0}^{\prime}\right)$ is weaker than that of $\left(H_{0}\right)$.

Since conditions $\left(H_{0}\right)$ and $\left(H_{0}^{\prime}\right)$ are all sufficient conditions, one of the interesting problems is whether the results still hold under the weaker condition. Now let us consider the following example.

Example 1. Consider the following system:

$$
\begin{aligned}
& x_{1}(n+1)=x_{1}(n) \exp [(0.6+0.3 \sin (n)) \\
& \quad-(1+0.3 \sin (n)) x_{1}(n)-(1+0.5 \sin (n)) x_{2}(n) \\
& \left.\quad-(1.1+\sin (n)) x_{1}(n) x_{2}(n)\right], \\
& x_{2}(n+1)=x_{2}(n) \exp [(0.6+0.3 \sin (n)) \\
& \quad-(2+0.6 \sin (n)) x_{1}(n)-(2+\sin (n)) x_{2}(n) \\
& \left.\quad-(2.2+2 \sin (n)) x_{1}(n) x_{2}(n)\right] .
\end{aligned}
$$

In this case

$$
\begin{aligned}
& r_{1}(n)=0.6+0.3 \sin (n), \\
& a_{1}(n)=1+0.3 \sin (n), \\
& b_{1}(n)=1+0.5 \sin (n), \\
& c_{1}(n)=1.1+\sin (n), \\
& r_{2}(n)=0.6+0.3 \sin (n), \\
& a_{2}(n)=2+0.6 \sin (n), \\
& b_{2}(n)=2+\sin (n), \\
& c_{2}(n)=2.2+2 \sin (n),
\end{aligned}
$$

By simple computation, one can see that

$$
\begin{aligned}
\frac{a_{2}^{l}}{a_{1}^{u}} & =\frac{1.4}{1.3}, \\
\frac{b_{2}^{l}}{b_{1}^{u}} & =\frac{1}{1.5}, \\
\frac{c_{2}^{l}}{c_{1}^{u}} & =\frac{0.2}{2.1}, \\
\frac{r_{2}^{u}}{r_{1}^{l}} & =\frac{0.9}{0.3}>\frac{0.2}{2.1}=\min \left\{\frac{a_{2}^{l}}{a_{1}^{u}}, \frac{b_{2}^{l}}{b_{1}^{u}}, \frac{c_{2}^{l}}{c_{1}^{u}}\right\}, \\
\frac{M\left[r_{2}\right]}{m\left[r_{1}\right]} & =1>\frac{0.2}{2.1}=\min \left\{\frac{a_{2}^{l}}{a_{1}^{u}}, \frac{b_{2}^{l}}{b_{1}^{u}}, \frac{c_{2}^{l}}{c_{1}^{u}}\right\},
\end{aligned}
$$

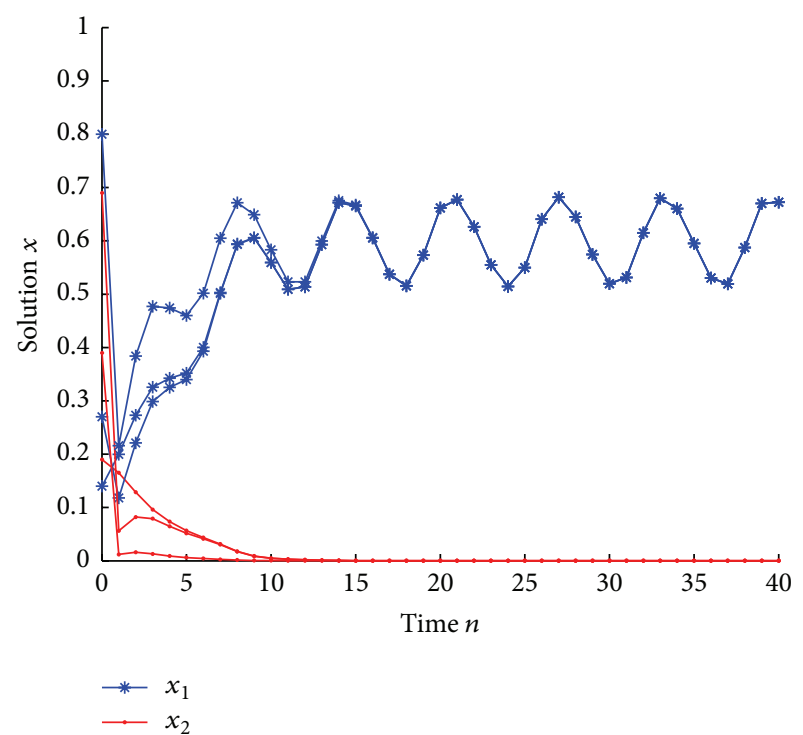

Figure 1: Dynamic behaviors of system (3) with initial values $\left(x_{1}(0), x_{2}(0)\right)=(0.14,0.19),(0.27,0.69)$, and $(0.8,0.39)$, respectively.

and (6) and (7) show that neither $\left(H_{0}\right)$ nor $\left(H_{0}^{\prime}\right)$ holds; hence one could not draw any conclusion about the dynamic behaviors of the system. However, Figure 1 shows species $x_{2}$ will be driven to extinction in this case. This motivates us to revisit the extinction property of system (2).

On the other hand, Gilpin and Ayala [21] conducted experiment on fruit fly dynamics to test the validity of 10 models of competitions. One of the models accounting best for the experimental results is given by

$$
\begin{aligned}
& \dot{x}_{1}(t)=r_{1} x_{1}(t)\left(1-\left(\frac{x_{1}(t)}{K_{1}}\right)^{\theta_{1}}-\alpha_{12} \frac{x_{2}(t)}{K_{2}}\right), \\
& \dot{x}_{2}(t)=r_{2} x_{2}(t)\left(1-\left(\frac{x_{2}(t)}{K_{2}}\right)^{\theta_{2}}-\alpha_{21} \frac{x_{1}(t)}{K_{1}}\right) .
\end{aligned}
$$

Fan and Wang [22] studied the dynamic behaviors of the following nonautonomous $n$-species Gilpin-Ayala competitive system:

$$
\begin{array}{r}
\dot{x}_{i}(t)=x_{i}(t)\left[b_{i}(t)-\sum_{j=1}^{n} a_{i j}(t)\left(x_{j}\left(t-\tau_{i j}(t)\right)\right)^{\theta_{i j}}\right], \\
i=1,2, \ldots, n,
\end{array}
$$

where $b_{i}(t), \tau_{i j}(t)$, and $a_{i j}(t), i, j=1,2, \ldots, n$, are continuous for $0 \leq t<+\infty$ and $\theta_{i j}$ are positive constants.

Chen et al. [23] studied a discrete $n$-species Gilpin-Ayala competitive system

$$
\begin{array}{r}
x_{i}(k+1)=x_{i}(k) \exp \left[b_{i}(k)-\sum_{j=1}^{n} a_{i j}(k)\left(x_{j}(k)\right)^{\theta_{i j}}\right], \\
i=1,2, \ldots, n,
\end{array}
$$


where $b_{i}(k), a_{i j}(k), i, j=1,2, \ldots, n$, are all positive sequences bounded above and below by positive constants. $\theta_{i j}$ are positive constants.

Recently, stimulated by the works of $[1,21,22]$, Chen et al. [24] proposed the following, a nonautonomous nonlinear competition system:

$$
\begin{aligned}
& \dot{x}_{1}(t)=x_{1}(t)\left[r_{1}(t)-a_{1}(t) x_{1}^{\alpha_{1}}(t)-b_{1}(t) x_{2}^{\alpha_{2}}(t)\right. \\
& \left.\quad-c_{1}(t) x_{1}^{\alpha_{1}}(t) x_{2}^{\alpha_{2}}(t)\right] \\
& \dot{x}_{2}(t)=x_{2}(t)\left[r_{2}(t)-a_{2}(t) x_{1}^{\alpha_{1}}(t)-b_{2}(t) x_{2}^{\alpha_{2}}(t)\right. \\
& \left.\quad-c_{2}(t) x_{1}^{\alpha_{1}}(t) x_{2}^{\alpha_{2}}(t)\right] .
\end{aligned}
$$

Chen et al. showed that if the coefficients of system (10) satisfy

$$
\limsup _{t \rightarrow+\infty} \frac{r_{2}(t)}{r_{1}(t)}<\liminf _{t \rightarrow+\infty}\left\{\frac{a_{2}(t)}{a_{1}(t)}, \frac{b_{2}(t)}{b_{1}(t)}, \frac{c_{2}(t)}{c_{1}(t)}\right\},
$$

the second species will be driven to extinction while the first one will stabilize at a certain solution of the system

$$
\dot{x}_{1}(t)=x_{1}(t)\left[r_{1}(t)-a_{1}(t) x_{1}^{\alpha_{1}}(t)\right] .
$$

Stimulated by the works of $[1-3,21-24]$, we propose the following a nonlinear discrete two species competition system:

$$
\begin{gathered}
x_{1}(n+1)=x_{1}(n) \exp \left[r_{1}(n)-a_{1}(n) x_{1}^{\alpha_{1}}(n)\right. \\
\left.-b_{1}(n) x_{2}^{\alpha_{2}}(n)-c_{1}(n) x_{1}^{\alpha_{1}}(n) x_{2}^{\alpha_{2}}(n)\right], \\
x_{2}(n+1)=x_{2}(n) \exp \left[r_{2}(n)-a_{2}(n) x_{1}^{\alpha_{1}}(n)\right. \\
\left.-b_{2}(n) x_{2}^{\alpha_{2}}(n)-c_{2}(n) x_{1}^{\alpha_{1}}(n) x_{2}^{\alpha_{2}}(n)\right] .
\end{gathered}
$$

We introduce the following assumptions:

$\left(H_{1}\right)\left\{r_{i}(n)\right\}$ are bounded sequence defined on $Z ;\left\{a_{i}(n)\right\}$, $\left\{b_{i}(n)\right\}$, and $\left\{c_{i}(n)\right\}, i=1,2$, are bounded nonnegative sequences defined on $Z ; \alpha_{i}, i=1,2$, are positive constants.

$\left(H_{2}\right)$ There exists positive integer $\omega$ such that for each $i=$ 1,2

$$
\liminf _{n \rightarrow \infty} \sum_{s=n}^{n+\omega-1} r_{i}(s)>0
$$

From the point of view of biology, we assume that $x_{i}(0)>0, i=1,2$; then system (14) has a positive solution $\left(x_{1}(n), x_{2}(n)\right)$ passing through $\left(x_{1}(0), x_{2}(0)\right)$.

The aim of this paper is, by developing the analysis technique of $\mathrm{Li}$ and Chen [2], Chen et al. [4, 24], and Xu et al. [6], to study the extinction property of system (14).

The organization of this paper is as follows. In Section 2, sufficient conditions for the permanence of system (14) are obtained. In Section 3, we study the extinction of species $x_{2}$. In Section 4 , we study the global stability of species $x_{1}$ when species $x_{2}$ is eventual extinction. Examples are presented in Section 5 to show the feasibility of our main results.

\section{Permanence}

Lemma 2 (see [26]). Assume that $\{x(n)\}$ satisfy $x(n)>0$ and

$$
x(n+1) \leq x(n) \exp \{a(n)-b(n) x(n)\}, \quad n \in N,
$$

where $a(n)$ and $b(n)$ are nonnegative sequences bounded above and below by positive constants. Then

$$
\lim _{n \rightarrow \infty} \sup x(n) \leq \frac{\exp \left\{a^{u}-1\right\}}{b^{l}} .
$$

Lemma 3 (see [26]). Assume that $\{x(n)\}$ satisfies

$$
x(n+1) \geq x(n) \exp \{a(n)-b(n) x(n)\}, \quad n \geq N_{0},
$$

limsup $\sup _{n \rightarrow \infty} x(n) \leq x^{*}$, and $x\left(N_{0}\right)>0$, where $a(n)$ and $b(n)$ are nonnegative sequences bounded above and below by positive constants and $N_{0} \in N$. Then

$$
\liminf _{n \rightarrow \infty} x(n) \geq \min \left\{\frac{a^{l}}{b^{u}} \exp \left\{a^{l}-b^{u} x^{*}\right\}, \frac{a^{l}}{b^{u}}\right\} .
$$

Lemma 4. Assume that $r_{1}^{l}>0$; every positive solution $\left(x_{1}(n)\right.$, $\left.x_{2}(n)\right)$ of system (14) satisfies

$$
\lim _{n \rightarrow \infty} \sup _{1}(n) \leq M_{1},
$$

where $M_{1}=\left(\exp \left\{\alpha_{1} r_{1}^{u}-1\right\} / \alpha_{1} a_{1}^{l}\right)^{1 / \alpha_{1}}$.

Proof. By the first equation of system (14), we have

$$
x_{1}(n) \leq x_{1}(n) \exp \left\{r_{1}(n)-a_{1}(n) x_{1}^{\alpha_{1}}(n)\right\} .
$$

Suppose $y_{1}(n)=x_{1}^{\alpha_{1}}(n)$; then $x_{1}(n)=\left[y_{1}(n)\right]^{1 / \alpha_{1}}$.

From (21), we have

$$
\begin{aligned}
& {\left[y_{1}(n+1)\right]^{1 / \alpha_{1}}} \\
& \quad \leq\left[y_{1}(n)\right]^{1 / \alpha_{1}} \exp \left\{r_{1}(n)-a_{1}(n) y_{1}(n)\right\} .
\end{aligned}
$$

That is,

$$
y_{1}(n+1) \leq y_{1}(n) \exp \left\{\alpha_{1} r_{1}(n)-\alpha_{1} a_{1}(n) y_{1}(n)\right\} .
$$

Applying Lemma 2 such that

$$
\limsup _{t \rightarrow+\infty} y_{1}(n) \leq \frac{\exp \left\{\alpha_{1} r_{1}^{u}-1\right\}}{\alpha_{1} a_{1}^{l}},
$$

hence

$$
\limsup _{t \rightarrow+\infty} x_{1}(n) \leq\left(\frac{\exp \left\{\alpha_{1} r_{1}^{u}-1\right\}}{\alpha_{1} a_{1}^{l}}\right)^{1 / \alpha_{1}} \stackrel{\text { def }}{=} M_{1} .
$$

Lemma 5. Assume that $r_{2}^{l}>0$; every positive solution $\left(x_{1}(n)\right.$, $\left.x_{2}(n)\right)$ of system (14) satisfies

$$
\lim _{n \rightarrow \infty} \sup _{2}(n) \leq M_{2},
$$

where $M_{2}=\left(\exp \left\{\alpha_{2} r_{2}^{u}-1\right\} / \alpha_{2} b_{2}^{l}\right)^{1 / \alpha_{2}}$. 
Proof. The proof of Lemma 5 is similar to that of Lemma 4, so we omit the detail here.

Lemma 6. Assume that

$$
\left(H_{3}\right) E_{1}>0
$$

holds; every positive solution $\left(x_{1}(n), x_{2}(n)\right)$ of system (14) satisfies

$$
\liminf _{n \rightarrow \infty} x_{1}(n) \geq m_{1},
$$

where $m_{1}=\left(E_{1} / E_{2}\right)^{1 / \alpha_{1}} \exp \left\{E_{1}-E_{2} M_{1}^{\alpha_{1}}\right\}, E_{1}=r_{1}^{l}-b_{1}^{u} M_{2}^{\alpha_{2}}$, and $E_{2}=a_{1}^{u}+c_{1}^{u} M_{2}^{\alpha_{2}}$.

Proof. In view of (26), for each $\varepsilon>0$, there exists a $N_{1}>0$ such that

$$
x_{2}(n) \leq M_{2}+\varepsilon, \quad \forall n \geq N_{1} .
$$

By the first equation of system (14), we have

$$
\begin{aligned}
x_{1} & (n+1) \geq x_{1}(n) \exp \left\{r_{1}(n)-a_{1}(n) x_{1}^{\alpha_{1}}(n)\right. \\
& \left.-b_{1}(n)\left(M_{2}+\varepsilon\right)^{\alpha_{2}}-c_{1}(n) x_{1}^{\alpha_{1}}(n)\left(M_{2}+\varepsilon\right)^{\alpha_{2}}\right\} \\
= & x_{1}(n) \exp \left\{r_{1}(n)-b_{1}(n)\left(M_{2}+\varepsilon\right)^{\alpha_{2}}\right. \\
& \left.-\left(a_{1}(n)+c_{1}(n)\left(M_{2}+\varepsilon\right)^{\alpha_{2}}\right) x_{1}^{\alpha_{1}}(n)\right\} .
\end{aligned}
$$

Suppose $y_{1}(n)=x_{1}^{\alpha_{1}}(n)$; then $x_{1}(n)=\left[y_{1}(n)\right]^{1 / \alpha_{1}}$.

From (28), we have

$$
\begin{aligned}
& {\left[y_{1}(n+1)\right]^{1 / \alpha_{1}} \geq\left[y_{1}(n)\right]^{1 / \alpha_{1}} \exp \left\{r_{1}(n)\right.} \\
& -b_{1}(n)\left(M_{2}+\varepsilon\right)^{\alpha_{2}} \\
& \left.\quad-\left(a_{1}(n)+c_{1}(n)\left(M_{2}+\varepsilon\right)^{\alpha_{2}}\right) y_{1}(n)\right\} .
\end{aligned}
$$

That is,

$$
\begin{aligned}
y_{1}(n+1) \geq y_{1}(n) \exp \left\{\alpha_{1} r_{1}(n)-\alpha_{1} b_{1}(n)\left(M_{2}+\varepsilon\right)^{\alpha_{2}}\right. & \left.-\left(\alpha_{1} a_{1}(n)+\alpha_{1} c_{1}(n)\left(M_{2}+\varepsilon\right)^{\alpha_{2}}\right) y_{1}(n)\right\} \\
\geq & y_{1}(n) \exp \left\{\alpha_{1} r_{1}^{l}-\alpha_{1} b_{1}^{u}\left(M_{2}+\varepsilon\right)^{\alpha_{2}}\right. \\
& \left.-\left(\alpha_{1} a_{1}^{u}+\alpha_{1} c_{1}^{u}\left(M_{2}+\varepsilon\right)^{\alpha_{2}}\right) y_{1}(n)\right\} \\
\stackrel{\operatorname{def}}{=} & y_{1}(n) \exp \left\{\alpha_{1} E_{1 \varepsilon}-\alpha_{1} E_{2 \varepsilon} y_{1}(n)\right\},
\end{aligned}
$$

where $E_{1 \varepsilon}=r_{1}^{l}-b_{1}^{u}\left(M_{2}+\varepsilon\right)^{\alpha_{2}}$ and $E_{2 \varepsilon}=a_{1}^{u}+c_{1}^{u}\left(M_{2}+\varepsilon\right)^{\alpha_{2}}$.

Applying Lemma 3 such that

$$
\begin{aligned}
& \liminf _{n \rightarrow+\infty} y_{1}(n) \\
& \geq \min \left\{\frac{E_{1}}{E_{2}} \exp \left\{\alpha_{1} E_{1}-\alpha_{1} E_{2} M_{1}^{\alpha_{1}}\right\}, \frac{E_{1}}{E_{2}}\right\},
\end{aligned}
$$

where $E_{1}=r_{1}^{l}-b_{1}^{u} M_{2}^{\alpha_{2}}$ and $E_{2}=a_{1}^{u}+c_{1}^{u} M_{2}^{\alpha_{2}}$.
Hence

$$
\begin{aligned}
& \liminf _{n \rightarrow+\infty} x_{1}(n) \\
& \geq \min \left\{\left(\frac{E_{1}}{E_{2}}\right)^{1 / \alpha_{1}} \exp \left\{E_{1}-E_{2} M_{1}^{\alpha_{1}}\right\},\left(\frac{E_{1}}{E_{2}}\right)^{1 / \alpha_{1}}\right\} .
\end{aligned}
$$

Note that

$$
\begin{aligned}
M_{1} & =\left(\frac{\exp \left\{\alpha_{1} r_{1}^{u}-1\right\}}{\alpha_{1} a_{1}^{l}}\right)^{1 / \alpha_{1}} \geq\left(\frac{\alpha_{1} r_{1}^{u}}{\alpha_{1} a_{1}^{l}}\right)^{1 / \alpha_{1}} \\
& =\left(\frac{r_{1}^{u}}{a_{1}^{l}}\right)^{1 / \alpha_{1}} .
\end{aligned}
$$

Thus

$$
r_{1}^{l}-a_{1}^{u} M_{1}^{\alpha_{1}} \leq 0
$$

And so

$$
E_{1}-E_{2} M_{1}^{\alpha_{1}} \leq 0
$$

Hence

$$
\liminf _{n \rightarrow+\infty} x_{1}(n) \geq\left(\frac{E_{1}}{E_{2}}\right)^{1 / \alpha_{1}} \exp \left\{E_{1}-E_{2} M_{1}^{\alpha_{1}}\right\} \stackrel{\text { def }}{=} m_{1} .
$$

Lemma 7. Assume that

$$
\left(H_{4}\right) F_{1}>0
$$

holds; every positive solution $\left(x_{1}(n), x_{2}(n)\right)$ of system (14) satisfies

$$
\liminf _{n \rightarrow \infty} x_{2}(n) \geq m_{2}
$$

where $m_{2}=\left(F_{1} / F_{2}\right)^{1 / \alpha_{2}} \exp \left\{F_{1}-F_{2} M_{2}^{\alpha_{2}}\right\}, F_{1}=r_{2}^{l}-a_{2}^{u} M_{1}^{\alpha_{1}}$, and $F_{2}=b_{2}^{u}+c_{2}^{u} M_{1}^{\alpha_{1}}$.

Proof. The proof of Lemma 7 is similar to that of Lemma 6, so we omit the detail here.

Lemma 8. Assume that $\left(H_{1}\right)-\left(H_{4}\right)$ hold; then system (14) is permanent. That is, for every solution $\left(x_{1}(n), x_{2}(n)\right)$ of system (14), one has

$$
m_{i} \leq \liminf _{n \rightarrow+\infty} x_{i}(n) \leq \limsup _{n \rightarrow+\infty} x_{i}(n) \leq M_{i}, \quad i=1,2 .
$$

\section{Extinction}

Theorem 9. Assume that $\left(H_{1}\right),\left(H_{2}\right)$ hold; assume further that
$\left(H_{5}\right) \lim \sup _{n \rightarrow \infty}\left(\sum_{s=n}^{n+\omega-1} r_{2}(s) / \sum_{s=n}^{n+\omega-1} r_{1}(s)\right)<$ $\lim \inf _{n \rightarrow \infty}\left\{a_{2}(n) / a_{1}(n), b_{2}(n) / b_{1}(n), c_{2}(n) / c_{1}(n) \mid n \in\right.$ $Z$ \}.

Let $\left(x_{1}(n), x_{2}(n)\right)$ be any positive solution of system (14); then $x_{2}(n) \rightarrow 0$ as $n \rightarrow+\infty$. 
Proof. By Lemma 2 we know that there exists $n_{1}>0$ such that

$$
x_{1}(n) \leq 2 M_{1}, \quad n \geq n_{1} .
$$

By $\left(H_{2}\right)$, there exist positive constants $\eta_{0}$ and $n_{2}>n_{1}$ such that

$$
\sum_{s=n}^{n+\omega-1} r_{i}(s) \geq \eta_{0}, \quad n \geq n_{2}
$$

By $\left(H_{5}\right)$, we can choose positive constants $\alpha, \beta$, and $\varepsilon$ such that

$$
\begin{gathered}
\limsup _{n \rightarrow \infty} \frac{\sum_{s=n}^{n+\omega-1} r_{2}(s)}{\sum_{s=n}^{n+\omega-1} r_{1}(s)}<\frac{\alpha}{\beta}-\varepsilon<\frac{\alpha}{\beta} \\
\quad<\liminf _{n \rightarrow \infty}\left\{\frac{a_{2}(n)}{a_{1}(n)}, \frac{b_{2}(n)}{b_{1}(n)}, \frac{c_{2}(n)}{c_{1}(n)}\right\} .
\end{gathered}
$$

Thus, there exists a $n_{3}>n_{2}>0$, such that for all $n \geq n_{3}$

$$
\begin{aligned}
\sum_{s=n}^{n+\omega-1}( & \left.\beta r_{2}(s)-\alpha r_{1}(s)\right)<-\varepsilon \beta \sum_{s=n}^{n+\omega-1} r_{1}(s)<-\varepsilon \beta \eta_{0} \\
& \alpha a_{1}(n)-\beta a_{2}(n)<0 \\
& \alpha b_{1}(n)-\beta b_{2}(n)<0 \\
& \alpha c_{1}(n)-\beta c_{2}(n)<0
\end{aligned}
$$

Let $V(n)=x_{1}^{-\alpha}(n) x_{2}^{\beta}(n)$; then

$$
\begin{aligned}
& \frac{V(n+1)}{V(n)}=\left[\frac{x_{1}(n+1)}{x_{1}(n)}\right]^{-\alpha}\left[\frac{x_{2}(n+1)}{x_{2}(n)}\right]^{\beta} \\
& =\exp \left\{-\alpha r_{1}(n)+\alpha a_{1}(n) x_{1}^{\alpha_{1}}(n)+\alpha b_{1}(n) x_{2}^{\alpha_{2}}(n)\right. \\
& \quad+\alpha c_{1}(n) x_{1}^{\alpha_{1}}(n) x_{2}^{\alpha_{2}}(n)+\beta r_{2}(n)-\beta a_{2}(n) x_{1}^{\alpha_{1}}(n) \\
& \left.\quad-\beta b_{2}(n) x_{2}^{\alpha_{2}}(n)-\beta c_{2}(n) x_{1}^{\alpha_{1}}(n) x_{2}^{\alpha_{2}}(n)\right\} \\
& =\exp \left\{\left(\beta r_{2}(n)-\alpha r_{1}(n)\right)\right. \\
& \quad+\left(\alpha a_{1}(n)-\beta a_{2}(n)\right) x_{1}^{\alpha_{1}}(n) \\
& \quad+\left(\alpha b_{1}(n)-\beta b_{2}(n)\right) x_{2}^{\alpha_{2}}(n) \\
& \left.\quad+\left(\alpha c_{1}(n)-\beta c_{2}(n)\right) x_{1}^{\alpha_{1}}(n) x_{2}^{\alpha_{2}}(n)\right\} .
\end{aligned}
$$

From (43) and (44), it follows that

$$
V(n+1) \leq V(n) \exp \left(\beta r_{2}(n)-\alpha r_{1}(n)\right), \quad n \geq n_{3} .
$$

For any $n \geq n_{3}$, we choose an integer $m \geq 0$ such that $n \in$ $\left[n_{3}+m \omega, n_{3}+(m+1) \omega\right)$. Integrating (46) from $n_{3}$ to $n-1$, from (43), we have

$$
\begin{aligned}
V(n) \leq V\left(n_{3}\right) \exp \left\{\sum_{s=n_{3}}^{n-1}\left(\beta r_{2}(s)-\alpha r_{1}(s)\right)\right\} \\
=V\left(n_{3}\right) \exp \left(\sum_{s=n_{3}}^{n_{3}+m \omega-1}+\sum_{s=n_{3}+m \omega-1}^{n-1}\right) \\
\cdot\left(\beta r_{2}(s)-\alpha r_{1}(s)\right) \\
\leq V\left(n_{3}\right) \exp \left\{-\varepsilon \beta \eta_{0} m+A_{1}\right\} \\
<V\left(n_{3}\right) \exp \left\{-\varepsilon \beta \eta_{0}\left(\frac{n-n_{3}}{\omega}-1\right)+A_{1}\right\} \\
=V\left(n_{3}\right) \exp \left\{-\frac{\varepsilon \beta \eta_{0} n}{\omega}+A_{1}^{*}\right\},
\end{aligned}
$$

where $A_{1}^{*}=\varepsilon \beta \eta_{0} n_{3} / \omega+\varepsilon \beta \eta_{0}+A_{1}$ and $A_{1}=\sup _{n \in Z} \mid \beta r_{2}(n)-$ $\alpha r_{1}(n) \mid \omega$.

(47) implies that

$$
\begin{aligned}
x_{2}(n)< & {\left[x_{1}^{-\alpha}\left(n_{3}\right) x_{2}^{\beta}\left(n_{3}\right)\left(2 M_{1}\right)^{\alpha} \exp \left\{A_{1}^{*}\right\}\right]^{1 / \beta} } \\
& \cdot \exp \left\{-\frac{\varepsilon \eta_{0} n}{\omega}\right\},
\end{aligned}
$$

for all $n \geq n_{3}$. Hence, $x_{2}(n) \rightarrow 0$ exponentially as $n \rightarrow+\infty$.

\section{Global Stability}

In Section 3, we prove that species $x_{2}$ will be driven to extinction if the conditions $\left(H_{1}\right),\left(H_{2}\right)$, and $\left(H_{5}\right)$ hold. Now we investigate the stability property of species $x_{1}$ under the same conditions.

Before we state the main result of this section, we first introduce some lemmas.

Lemma 10. Assume that $\left(H_{1}\right),\left(H_{2}\right),\left(H_{5}\right)$, and $r_{1}^{l}>0$ hold; let $\left(x_{1}(n), x_{2}(n)\right)$ be any positive solution of system (14); then

$$
m_{1}^{\prime} \leq \liminf _{n \rightarrow \infty} x_{1}(n) \leq \limsup _{n \rightarrow \infty} x_{1}(n) \leq M_{1},
$$

where $m_{1}^{\prime}=\left(r_{1}^{l} / a_{1}^{u}\right)^{1 / \alpha_{1}} \exp \left\{r_{1}^{l}-a_{1}^{u} M_{1}^{\alpha_{1}}\right\}$.

Proof. Under the assumption conditions $\left(H_{1}\right),\left(H_{2}\right)$, and $\left(H_{5}\right)$, it follows from Theorem 9 that

$$
\lim _{n \rightarrow \infty} x_{2}(n)=0 .
$$

From Lemma 4, we have

$$
\limsup _{n \rightarrow \infty} x_{1}(n) \leq M_{1} \text {. }
$$

By Lemma 10, it is enough to show that

$$
\liminf _{n \rightarrow \infty} x_{1}(n) \geq m_{1}^{\prime} \text {. }
$$


In view of (50) and (51), for each $\varepsilon>0$, there exists an integer $n_{0} \in Z$ such that

$$
\begin{aligned}
& x_{1}(n) \leq M_{1}+\varepsilon, \\
& x_{2}(n) \leq \varepsilon,
\end{aligned}
$$

$$
\forall n \geq n_{0} .
$$

We consider the following two cases.

Case 1 . We assume that there exists an $l_{0} \geq n_{0}$ such that $x_{1}\left(l_{0}+\right.$ 1) $\leq x_{1}\left(l_{0}\right)$. Note that for $n \geq l_{0}$

$$
\begin{aligned}
& x_{1}(n+1)=x_{1}(n) \exp \left[r_{1}(n)-a_{1}(n) x_{1}^{\alpha_{1}}(n)\right. \\
& \left.\quad-b_{1}(n) x_{2}^{\alpha_{2}}(n)-c_{1}(n) x_{1}^{\alpha_{1}}(n) x_{2}^{\alpha_{2}}(n)\right] \\
& \geq x_{1}(n) \exp \left[r_{1}^{l}-a_{1}^{u} x_{1}^{\alpha_{1}}(n)-b_{1}^{u} \varepsilon^{\alpha_{2}}\right. \\
& \left.\quad-c_{1}^{u}\left(M_{1}+\varepsilon\right)^{\alpha_{1}} \varepsilon^{\alpha_{2}}\right] .
\end{aligned}
$$

In particular, with $n=l_{0}$, we obtain

$$
r_{1}^{l}-a_{1}^{u} x_{1}^{\alpha_{1}}\left(l_{0}\right)-b_{1}^{u} \varepsilon^{\alpha_{2}}-c_{1}^{u}\left(M_{1}+\varepsilon\right)^{\alpha_{1}} \varepsilon^{\alpha_{2}} \leq 0,
$$

which implies that

$$
x_{1}\left(l_{0}\right) \geq\left(\frac{r_{1}^{l}-\left(b_{1}^{u}+c_{1}^{u}\left(M_{1}+\varepsilon\right)^{\alpha_{1}}\right) \varepsilon^{\alpha_{2}}}{a_{1}^{u}}\right)^{1 / \alpha_{1}} .
$$

From (54) and (56), it follows that

$$
\begin{aligned}
& x_{1}\left(l_{0}+1\right) \geq\left(\frac{r_{1}^{l}-\left(b_{1}^{u}+c_{1}^{u}\left(M_{1}+\varepsilon\right)^{\alpha_{1}}\right) \varepsilon^{\alpha_{2}}}{a_{1}^{u}}\right)^{1 / \alpha_{1}} \\
& \cdot \exp \left[r_{1}^{l}-a_{1}^{u} x_{1}^{\alpha_{1}}\left(l_{0}\right)-b_{1}^{u} \varepsilon^{\alpha_{2}}-c_{1}^{u}\left(M_{1}+\varepsilon\right)^{\alpha_{1}} \varepsilon^{\alpha_{2}}\right] \\
& \geq\left(\frac{r_{1}^{l}-\left(b_{1}^{u}+c_{1}^{u}\left(M_{1}+\varepsilon\right)^{\alpha_{1}}\right) \varepsilon^{\alpha_{2}}}{a_{1}^{u}}\right)^{1 / \alpha_{1}} \exp \left[r_{1}^{l}\right. \\
& \left.-a_{1}^{u}\left(M_{1}+\varepsilon\right)^{\alpha_{1}}-b_{1}^{u} \varepsilon^{\alpha_{2}}-c_{1}^{u}\left(M_{1}+\varepsilon\right)^{\alpha_{1}} \varepsilon^{\alpha_{2}}\right] .
\end{aligned}
$$

Let

$$
\begin{aligned}
x_{1 \varepsilon} & =\left(\frac{r_{1}^{l}-\left(b_{1}^{u}+c_{1}^{u}\left(M_{1}+\varepsilon\right)^{\alpha_{1}}\right) \varepsilon^{\alpha_{2}}}{a_{1}^{u}}\right)^{1 / \alpha_{1}} \exp \left[r_{1}^{l}\right. \\
& \left.-a_{1}^{u}\left(M_{1}+\varepsilon\right)^{\alpha_{1}}-b_{1}^{u} \varepsilon^{\alpha_{2}}-c_{1}^{u}\left(M_{1}+\varepsilon\right)^{\alpha_{1}} \varepsilon^{\alpha_{2}}\right] .
\end{aligned}
$$

Note that

$$
\begin{aligned}
M_{1} & =\left(\frac{\exp \left\{\alpha_{1} r_{1}^{u}-1\right\}}{\alpha_{1} a_{1}^{l}}\right)^{1 / \alpha_{1}} \geq\left(\frac{\alpha_{1} r_{1}^{u}}{\alpha_{1} a_{1}^{l}}\right)^{1 / \alpha_{1}} \\
& \geq\left(\frac{r_{1}^{u}}{a_{1}^{l}}\right)^{1 / \alpha_{1}}
\end{aligned}
$$

and thus $r_{1}^{l}-a_{1}^{u} M_{1}^{\alpha_{1}} \leq 0$; also, for arbitrary $\varepsilon$,

$$
r_{1}^{l}-a_{1}^{u}\left(M_{1}+\varepsilon\right)^{\alpha_{1}}-b_{1}^{u} \varepsilon^{\alpha_{2}}-c_{1}^{u}\left(M_{1}+\varepsilon\right)^{\alpha_{1}} \varepsilon^{\alpha_{2}} \leq 0,
$$

or

$$
\left(\frac{r_{1}^{l}-\left(b_{1}^{u}+c_{1}^{u}\left(M_{1}+\varepsilon\right)^{\alpha_{1}}\right) \varepsilon^{\alpha_{2}}}{a_{1}^{u}}\right)^{1 / \alpha_{1}} \geq x_{1 \varepsilon} .
$$

We claim that

$$
x_{1}(n) \geq x_{1 \varepsilon}, \quad \forall n \geq l_{0} .
$$

By way of contradiction, assume that there exists a $p_{0}>l_{0}$ such that $x_{1}\left(p_{0}\right)<x_{1 \varepsilon}$. Then $p_{0} \geq l_{0}+2$. Let $\tilde{p}_{0} \geq l_{0}+2$ be the smallest integer such that $x_{1}\left(\widetilde{p}_{0}\right)<x_{1 \varepsilon}$. Then $x_{1}\left(\widetilde{p}_{0}-1\right)>$ $x_{1}\left(\widetilde{p}_{0}\right)$. The above argument produces that $x_{1}\left(\widetilde{p}_{0}\right) \geq x_{1 \varepsilon}$, a contradiction. This proves the claim.

Case 2. We assume that $x_{1}(n+1)>x_{1}(n)$ for $n \geq n_{0}$; then $\lim _{n \rightarrow \infty} x_{1}(n)=\underline{x}_{1}$. We claim that

$$
\underline{x}_{1} \geq\left(\frac{r_{1}^{l}-\left(b_{1}^{u}+c_{1}^{u}\left(M_{1}+\varepsilon\right)^{\alpha_{1}}\right) \varepsilon^{\alpha_{2}}}{a_{1}^{u}}\right)^{1 / \alpha_{1}} .
$$

By way of contradiction, assume that

$$
\underline{x}_{1}<\left(\frac{r_{1}^{l}-\left(b_{1}^{u}+c_{1}^{u}\left(M_{1}+\varepsilon\right)^{\alpha_{1}}\right) \varepsilon^{\alpha_{2}}}{a_{1}^{u}}\right)^{1 / \alpha_{1}} .
$$

Taking limit in the first equation in system (14) gives

$$
\begin{aligned}
\lim _{n \rightarrow \infty}\left(r_{1}(n)-a_{1}(n) x_{1}^{\alpha_{1}}(n)-b_{1}(n) x_{2}^{\alpha_{2}}(n)\right. \\
\left.-c_{1}(n) x_{1}^{\alpha_{1}}(n) x_{2}^{\alpha_{2}}(n)\right)=0,
\end{aligned}
$$

which is a contradiction since

$$
\begin{aligned}
\lim _{n \rightarrow \infty} & \left(r_{1}(n)-a_{1}(n) x_{1}^{\alpha_{1}}(n)-b_{1}(n) x_{2}^{\alpha_{2}}(n)\right. \\
& \left.-c_{1}(n) x_{1}^{\alpha_{1}}(n) x_{2}^{\alpha_{2}}(n)\right) \geq r_{1}^{l}-a_{1}^{u} \underline{x}_{1}^{\alpha_{1}}-b_{1}^{u} \varepsilon^{\alpha_{2}} \\
& -c_{1}^{u}\left(M_{1}+\varepsilon\right)^{\alpha_{1}} \varepsilon^{\alpha_{2}}>0 .
\end{aligned}
$$

This proves the claim; then we have

$$
\liminf _{n \rightarrow \infty} x_{1}(n)=\lim _{n \rightarrow \infty} x_{1}(n)=\underline{x}_{1} \geq x_{1 \varepsilon} .
$$

Combining Case 1 and Case 2, we see that

$$
\liminf _{n \rightarrow \infty} x_{1}(n) \geq x_{1 \varepsilon} .
$$

Setting $\varepsilon \rightarrow 0$, note that

$$
\lim _{\varepsilon \rightarrow 0} x_{1 \varepsilon}=\left(\frac{r_{1}^{l}}{a_{1}^{u}}\right)^{1 / \alpha_{1}} \exp \left\{r_{1}^{l}-a_{1}^{u} M_{1}^{\alpha_{1}}\right\} \stackrel{\text { def }}{=} m_{1}^{\prime} .
$$

Now, we can easily see that (52) holds. This completes the proof of Lemma 10. 
We consider a discrete equation

$$
\begin{aligned}
& x(n+1)=x(n) \exp \left(r_{1}(n)-a_{1}(n) x^{\alpha_{1}}(n)\right), \\
& n \in N \text {, }
\end{aligned}
$$

where $\left\{r_{1}(n)\right\}$ and $\left\{a_{1}(n)\right\}$ are bounded nonnegative sequences; similarly to the proof of Lemma 10, we can obtain the following lemma.

Lemma 11. For any positive solution $\{x(n)\}$ of (70), one has

$$
m \leq \liminf _{n \rightarrow \infty} x(n) \leq \lim _{n \rightarrow \infty} \sup x(n) \leq M,
$$

where $M=\left(\exp \left\{\alpha_{1} r_{1}^{u}-1\right\} / \alpha_{1} a_{1}^{l}\right)^{1 / \alpha_{1}}$ and $m=$ $\left(r_{1}^{l} / a_{1}^{u}\right)^{1 / \alpha_{1}} \exp \left\{r_{1}^{l}-a_{1}^{u} M^{\alpha_{1}}\right\}$.

Now, we state the main result of this section.

Theorem 12. Assume that $\left(H_{1}\right),\left(H_{2}\right),\left(H_{5}\right)$, and $r_{1}^{l}>0$ hold; assume further that

$$
\frac{a_{1}^{u}}{a_{1}^{l}} \exp \left(\alpha_{1} r_{1}^{u}-1\right)<2
$$

then for any positive solution $\left(x_{1}(n), x_{2}(n)\right)$ of system (14) and any positive solution $\{x(n)\}$ of system (70), one has

$$
\begin{array}{r}
\lim _{n \rightarrow \infty}\left(x_{1}(n)-x(n)\right)=0, \\
\lim _{n \rightarrow \infty} x_{2}(n)=0 .
\end{array}
$$

Proof. Since $\left(H_{1}\right),\left(H_{2}\right)$, and $\left(H_{5}\right)$ hold, it follows from Theorem 12 that

$$
\lim _{n \rightarrow \infty} x_{2}(n)=0 .
$$

To prove $\lim _{n \rightarrow \infty}\left(x_{1}(n)-x(n)\right)=0$, let

$$
x_{1}(n)=x(n) \exp (y(n)) .
$$

It follows from the first equation of system (14) and (74) that

$$
\begin{aligned}
y(n+1)= & y(n)-a_{1}(n) x^{\alpha_{1}}(n)\left(\exp \left(\alpha_{1} y(n)\right)-1\right) \\
& -b_{1}(n) x_{2}^{\alpha_{2}}(n)-c_{1}(n) x_{1}^{\alpha_{1}}(n) x_{2}^{\alpha_{2}}(n) .
\end{aligned}
$$

Using the Mean Value Theorem, we get

$$
\begin{aligned}
\exp \left(\alpha_{1} y(n)\right)-1=\alpha_{1} \exp (\theta(n)) y(n) & \\
\theta(n) & \in\left(0, \alpha_{1} y(n)\right) .
\end{aligned}
$$

Then the first equation of system (14) is equivalent to

$$
\begin{aligned}
y(n+1)= & \left(1-\alpha_{1} a_{1}(n) x^{\alpha_{1}}(n) \exp (\theta(n))\right) y(n) \\
& -\left(b_{1}(n)+c_{1}(n) x_{1}^{\alpha_{1}}(n)\right) x_{2}^{\alpha_{2}}(n),
\end{aligned}
$$

where $\theta(n) \in\left(0, \alpha_{1} y(n)\right)$.

To complete the proof, it suffices to show that

$$
\lim _{n \rightarrow \infty} y(n)=0 \text {. }
$$

We first assume that

$$
\lambda=\max \left\{\left|1-\alpha_{1} a_{1}^{u} M_{1}^{\alpha_{1}}\right|,\left|1-\alpha_{1} a_{1}^{l} m^{\alpha_{1}}\right|\right\}<1,
$$

and then we can choose positive constant $\varepsilon>0$ small enough such that

$$
\begin{aligned}
& \lambda_{\varepsilon} \\
& \quad=\max \left\{\left|1-\alpha_{1} a_{1}^{u}\left(M_{1}+\varepsilon\right)^{\alpha_{1}}\right|,\left|1-\alpha_{1} a_{1}^{l}(m-\varepsilon)^{\alpha_{1}}\right|\right\} \\
& \quad<1 .
\end{aligned}
$$

For above $\varepsilon$, according to Lemmas 10 and 11 and (73), there exists an integer $n_{0} \in Z$ such that

$$
\begin{aligned}
& m-\varepsilon \leq x(n), \\
& x_{1}(n) \leq M_{1}+\varepsilon, \\
& x_{2}(n) \leq \varepsilon,
\end{aligned}
$$

for $n \geq n_{0}$.

It follows from (81) that

$$
\begin{aligned}
b_{1}(n)+c_{1}(n) x_{1}^{\alpha_{1}}(n) \leq b_{1}^{u}+c_{1}^{u}\left(M_{1}+\varepsilon\right) \stackrel{\alpha_{1}}{\stackrel{\text { def }}{=}} M_{\mathcal{\varepsilon}}, \\
\\
\text { for } n \geq n_{0} .
\end{aligned}
$$

Note that $\theta(n) \in\left(0, \alpha_{1} y(n)\right)$ implies that $\alpha_{1} x^{\alpha_{1}}(n) \exp (\theta(n))$ lies between $\alpha_{1} x^{\alpha_{1}}(n)$ and $\alpha_{1} x_{1}^{\alpha_{1}}(n)$. From (77) and (80)-(82), we get

$$
\begin{aligned}
& |y(n+1)| \\
& \quad \leq \max \left\{\left|1-\alpha_{1} a_{1}^{u}\left(M_{1}+\varepsilon\right)^{\alpha_{1}}\right|,\left|1-\alpha_{1} a_{1}^{l}(m-\varepsilon)^{\alpha_{1}}\right|\right\} \\
& \cdot|y(n)|+\left(b_{1}^{u}+c_{1}^{u}\left(M_{1}+\varepsilon\right)^{\alpha_{1}}\right) \varepsilon^{\alpha_{2}}=\lambda_{\varepsilon}|y(n)| \\
& \quad+M_{\varepsilon} \varepsilon^{\alpha_{2}}, \quad \text { for } n \geq n_{0} .
\end{aligned}
$$

This implies that

$$
|y(n)| \leq \lambda_{\varepsilon}^{n-n_{0}}\left|y\left(n_{0}\right)\right|+\frac{1-\lambda_{\varepsilon}^{n-n_{0}}}{1-\lambda_{\varepsilon}} M_{\varepsilon} \varepsilon^{\alpha_{2}},
$$

for $n \geq n_{0}$.

Since $\lambda_{\varepsilon}<1$ and $\varepsilon$ is arbitrary small, we obtain $\lim _{n \rightarrow \infty} y(n)=$ 0 ; it means that (78) holds when $\lambda<1$.

Note that

$$
1-\alpha_{1} a_{1}^{u} M_{1}^{\alpha_{1}} \leq 1-\alpha_{1} a_{1}^{l} m^{\alpha_{1}}<1,
$$

and thus, $\lambda<1$ is equivalent to

$$
1-\alpha_{1} a_{1}^{u} M_{1}^{\alpha_{1}}>-1
$$

or

$$
\alpha_{1} a_{1}^{u} M_{1}^{\alpha_{1}}=\frac{a_{1}^{u}}{a_{1}^{l}} \exp \left(\alpha_{1} r_{1}^{u}-1\right)<2 .
$$

Now, we can conclude that (78) is satisfied as $\left(\mathrm{H}_{6}\right)$ holds, and so $\lim _{n \rightarrow \infty}\left(x_{1}(n)-x(n)\right)=0$. This completes the proof of Theorem 12. 
As a direct corollary of Theorems 9 and 12, for system (2), we have the following result.

Corollary 13. Assume that $\left(H_{1}\right),\left(H_{2}\right),\left(H_{5}\right)$, and $r_{1}^{l}>0$ hold; assume further that

$$
\frac{a_{1}^{u}}{a_{1}^{l}} \exp \left(r_{1}^{u}-1\right)<2
$$

then for any positive solution $\left(x_{1}(n), x_{2}(n)\right)$ of system (2) and any positive solution $\{x(n)\}$ of

$$
x_{1}(n+1)=x_{1}(n) \exp \left\{r_{1}(n)-a_{1}(n) x_{1}(n)\right\}
$$

one has

$$
\begin{array}{r}
\lim _{n \rightarrow \infty}\left(x_{1}(n)-x(n)\right)=0, \\
\lim _{n \rightarrow \infty} x_{2}(n)=0 .
\end{array}
$$

\section{Examples}

The following examples show the feasibility of our main results.

Example 14. Now let us consider Example 1; in this case, one can easily check that

$$
\begin{aligned}
\liminf _{n \rightarrow \infty} \sum_{s=n}^{n+\omega-1} r_{i}(s)>0, \\
\limsup _{n \rightarrow \infty} \frac{\sum_{s=n}^{n+\omega-1} r_{2}(s)}{\sum_{s=n}^{n+\omega-1} r_{1}(s)}=1, \\
\frac{a_{2}(n)}{a_{1}(n)}=\frac{b_{2}(n)}{b_{1}(n)}=\frac{c_{2}(n)}{c_{1}(n)}=2 ;
\end{aligned}
$$

hence

$$
\begin{aligned}
& \limsup _{n \rightarrow \infty} \frac{\sum_{s=n}^{n+\omega-1} r_{2}(s)}{\sum_{s=n}^{n+\omega-1} r_{1}(s)}<2 \\
& \quad=\liminf _{n \rightarrow \infty}\left\{\frac{a_{2}(n)}{a_{1}(n)}, \frac{b_{2}(n)}{b_{1}(n)}, \frac{c_{2}(n)}{c_{1}(n)}\right\} .
\end{aligned}
$$

Also

$$
\frac{a_{1}^{u}}{a_{1}^{l}} \exp \left(\alpha_{1} r_{1}^{u}-1\right)=\frac{1.3}{0.7} \exp (0.9-1) \approx 1.680<2 .
$$

Equations (91)-(93) show that all the conditions of Corollary 13 hold; then species $x_{2}$ will be driven to extinction while species $x_{1}$ will be globally attractive with any positive solution of the following discrete equation:

$$
\begin{aligned}
& x_{1}(n+1)=x_{1}(n) \\
& \quad \cdot \exp \left[0.6+0.3 \sin (n)-(1+0.3 \sin (n)) x_{1}(n)\right] .
\end{aligned}
$$

Example 15. Consider the following system:

$$
\begin{aligned}
& x_{1}(n+1)=x_{1}(n) \exp \left[0.6-(1.5+0.2 \sin (n)) x_{1}^{2}(n)\right. \\
& -(1+0.5 \sin (n)) x_{2}^{1 / 2}(n) \\
& \left.-(1.1+\sin (n)) x_{1}^{2}(n) x_{2}^{1 / 2}(n)\right], \\
& x_{2}(n+1)=x_{2}(n) \exp \left[0.3-(1.4+0.5 \sin (n)) x_{1}^{2}(n)\right. \\
& \left.-(1.3+0.5 \sin (n)) x_{2}^{1 / 2}(n)-1.2 x_{1}^{2}(n) x_{2}^{1 / 2}(n)\right] .
\end{aligned}
$$

In this case, corresponding to system $(2), r_{1}(n)=0.6, r_{2}(n)=$ $0.3, a_{1}(n)=1.5+0.2 \sin (n), b_{1}(n)=1+0.5 \sin (n), c_{1}(n)=$ $1.1+\sin (n), a_{2}(n)=1.4+0.5 \sin (n), b_{2}(n)=1.3+0.5 \sin (n)$, $c_{2}(n)=1.2, \alpha_{1}=2$, and $\alpha_{2}=1 / 2$. By simple computation, one can see that

$$
\begin{aligned}
& \frac{a_{2}(n)}{a_{1}(n)} \geq \frac{0.9}{1.7}, \\
& \frac{b_{2}(n)}{b_{1}(n)} \geq \frac{0.8}{1.5}, \\
& \frac{c_{2}(n)}{c_{1}(n)} \geq \frac{1.2}{2.1},
\end{aligned}
$$$$
\liminf _{n \rightarrow \infty} \sum_{s=n}^{n+\omega-1} r_{i}(s)>0,
$$$$
\limsup _{n \rightarrow \infty} \frac{\sum_{s=n}^{n+\omega-1} r_{2}(s)}{\sum_{s=n}^{n+\omega-1} r_{1}(s)}=\frac{1}{2},
$$$$
\leq \liminf _{n \rightarrow \infty}\left\{\frac{a_{2}(n)}{a_{1}(n)}, \frac{b_{2}(n)}{b_{1}(n)}, \frac{c_{2}(n)}{c_{1}(n)}\right\},
$$$$
\frac{a_{1}^{u}}{a_{1}^{l}} \exp \left(\alpha_{1} r_{1}^{u}-1\right) \leq \frac{1.7}{1.3} \exp (2 \times 0.6-1) \approx 1.5972
$$

$$
<2 \text {. }
$$

Equations (97) and (99) show that all the conditions of Theorem 12 hold; thus species $x_{2}$ is driven to extinction while species $x_{1}$ is asymptotic to any positive solution of

$$
\begin{aligned}
& x(n+1) \\
& \quad=x(n) \exp \left(0.6-(1.5+0.2 \sin (n)) x^{2}(n)\right) .
\end{aligned}
$$

Figure 2 shows the dynamic behaviors of system (95).

\section{Conclusion}

In this paper, we consider a nonlinear discrete two species competition system with the effect of toxic substances. In 


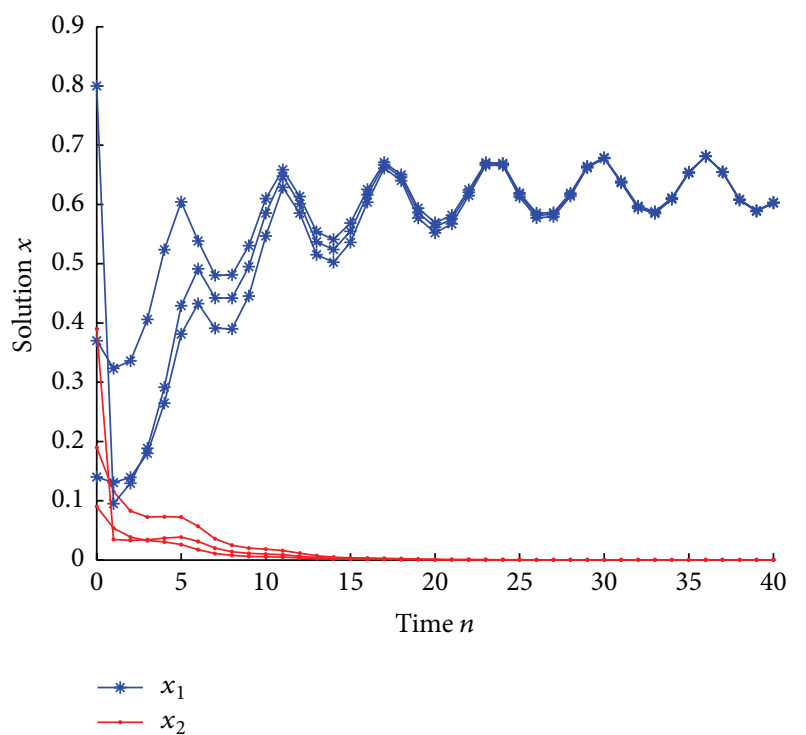

FIGURE 2: Dynamic behaviors of system (95) with initial values $\left(x_{1}(0), x_{2}(0)\right)=(0.14,0.19),(0.27,0.69)$, and $(0.8,0.39)$, respectively.

Theorem 9, by constructing a suitable Lyapunov-type function, we obtain a set of sufficient conditions which ensure species $x_{2}$ will be driven to extinction. Our results improve and generalize Theorem 2.1 of [2] and Theorem 1.1 of [3].

\section{Competing Interests}

The authors declare that there are no competing interests.

\section{Acknowledgments}

The research was supported by the Natural Science Foundation of Fujian Province (2015J010121, 2015J01019).

\section{References}

[1] Z. Li and F. Chen, "Extinction in two dimensional nonautonomous Lotka-Volterra systems with the effect of toxic substances," Applied Mathematics and Computation, vol. 182, no. 1, pp. 684-690, 2006.

[2] Z. Li and F. Chen, "Extinction in two dimensional discrete Lotka-Volterra competitive systems with the effect of toxic substances," Dynamics of Continuous Discrete and Implusive System Series B, vol. 15, no. 1, pp. 165-178, 2008.

[3] Y. Guo, X. Li, and E. Zhang, "Extinction and stability for a discrete Lotka-Volterra system," Journal of Biomathematics, vol. 26, no. 4, pp. 593-600, 2011.

[4] L. Chen and F. Chen, "Extinction in a discrete Lotka-Volterra competitive system with the effect of toxic substances and feedback controls," International Journal of Biomathematics, vol. 8, no. 1, Article ID 1550012, 13 pages, 2015.

[5] J. Xu, Z. Teng, and H. Jiang, "Permanence and global attractivity for discrete nonautonomous two-species Lotka-Volterra competitive system with delays and feedback controls," Periodica Mathematica Hungarica, vol. 63, no. 1, pp. 19-45, 2011.
[6] J. Xu, Z. Teng, and S. Gao, "Almost sufficient and necessary conditions for permanence and extinction of nonautonomous discrete logistic systems with time-varying delays and feedback control," Applications of Mathematics, vol. 56, no. 2, pp. 207-225, 2011.

[7] A. Muhammadhaji, Z. Teng, and L. Nie, "Permanence in nonautonomous discrete Lotka-Volterra n-species competitive system with pure-delays and feedback control," International Journal of Mathematics, vol. 24, no. 7, Article ID 1350053, 17 pages, 2013.

[8] Z. Liu, J. Hui, and J. Wu, "Permanence and partial extinction in an impulsive delay competitive system with the effect of toxic substances," Journal of Mathematical Chemistry, vol. 46, no. 4, pp. 1213-1231, 2009.

[9] R. Tan, Z. Li, Q. Wang, and Z. Liu, "Positive periodic solutions of a periodic discrete competitive system subject to feedback controls," Journal of Applied Mathematics, vol. 2014, Article ID 927626, 10 pages, 2014.

[10] Q. Wang, Z. Liu, and Z. Li, "Positive almost periodic solutions for a discrete competitive system subject to feedback controls," Journal of Applied Mathematics, vol. 2013, Article ID 429163, 14 pages, 2013.

[11] C. Liu and Y. Li, "Global stability analysis of a nonautonomous stage-structured competitive system with toxic effect and double maturation delays," Abstract and Applied Analysis, vol. 2014, Article ID 689573, 15 pages, 2014.

[12] Z. Liu and L. Chen, "Periodic solution of a two-species competitive system with toxicant and birth pulse," Chaos, Solitons \& Fractals, vol. 32, no. 5, pp. 1703-1712, 2007.

[13] S. Yu, "Permanence for a discrete competitive system with feedback controls," Communications in Mathematical Biology and Neuroscience, vol. 2015, article 16, 11 pages, 2015.

[14] D. Wang, "Permanence of a Gilpin-Ayala competition system with infinite delays and single feedback control," Communications in Mathematical Biology and Neuroscience, vol. 2015, article 9, 9 pages, 2015.

[15] L. Li and R. Wu, "Extinction of a delay differential equation model of plankton allelopathy," Communications in Mathematical Biology and Neuroscience, vol. 2015, article 13, 14 pages, 2015.

[16] R. P. Gupta, M. Banerjee, and P. Chandra, "The dynamics of two-species allelopathic competition with optimal harvesting," Journal of Biological Dynamics, vol. 6, no. 2, pp. 674-694, 2012.

[17] A. Muhammadhaji, R. Mahemuti, and Z. Teng, "Periodic solutions for $n$-species Lotka-Volterra competitive systems with pure delays," Chinese Journal of Mathematics, vol. 2015, Article ID 856959, 11 pages, 2015.

[18] F. Montes de Oca and M. Vivas, "Extinction in two dimensional Lotka-Volterra system with infinite delay," Nonlinear Analysis: Real World Applications, vol. 7, no. 5, pp. 1042-1047, 2006.

[19] Z. Hou, "On permanence of all subsystems of competitive Lotka-Volterra systems with delays," Nonlinear Analysis. Real World Applications, vol. 11, no. 5, pp. 4285-4301, 2010.

[20] Z. Hou, "Permanence and extinction in competitive LotkaVolterra systems with delays," Nonlinear Analysis. Real World Applications. An International Multidisciplinary Journal, vol. 12, no. 4, pp. 2130-2141, 2011.

[21] M. E. Gilpin and F. J. Ayala, "Global models of growth and competition," Proceedings of the National Academy of Sciences of the United States of America, vol. 70, no. 12, pp. 3590-3593, 1973. 
[22] M. Fan and K. Wang, "Global periodic solutions of a generalized $n$-species Gilpin-Ayala competition model," Computers \& Mathematics with Applications, vol. 40, no. 10-11, pp. 1141-1151, 2000.

[23] F. Chen, L. Wu, and Z. Li, "Permanence and global attractivity of the discrete Gilpin-Ayala type population model," Computers \& Mathematics with Applications, vol. 53, no. 8, pp. 1214-1227, 2007.

[24] F. Chen, X. Xie, Z. Miao, and L. Pu, "Extinction in two species nonautonomous nonlinear competitive system," Applied Mathematics and Computation, vol. 274, no. 1, pp. 119-124, 2016.

[25] R. Wu and L. Li, "Extinction of a reaction-diffusion model of plankton allelopathy with nonlocal delays," Communications in Mathematical Biology and Neuroscience, vol. 2015, article 8, 19 pages, 2015.

[26] F. Chen, "Permanence and global attractivity of a discrete multispecies Lotka-Volterra competition predator-prey systems," Applied Mathematics and Computation, vol. 182, no. 1, pp. 3-12, 2006. 


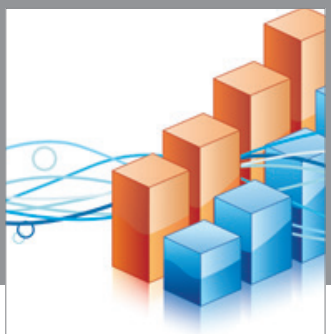

Advances in

Operations Research

vatem alat4

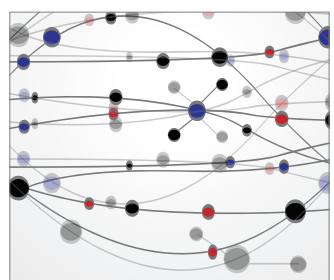

\section{The Scientific} World Journal
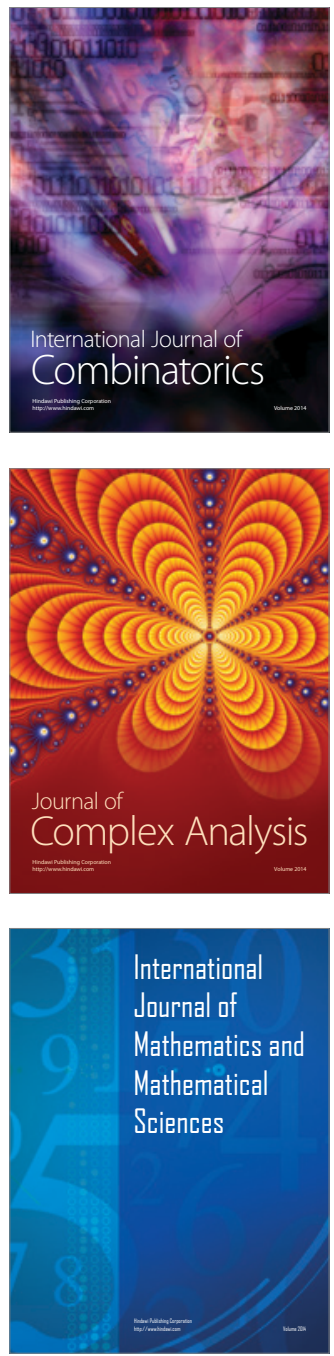
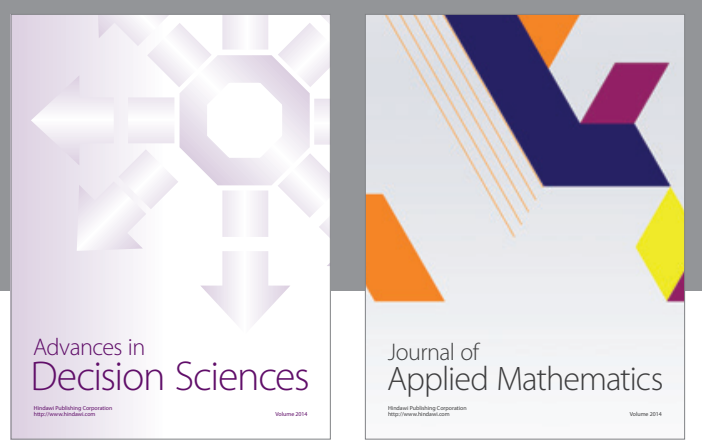

Algebra

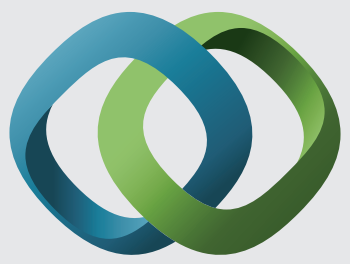

\section{Hindawi}

Submit your manuscripts at

http://www.hindawi.com
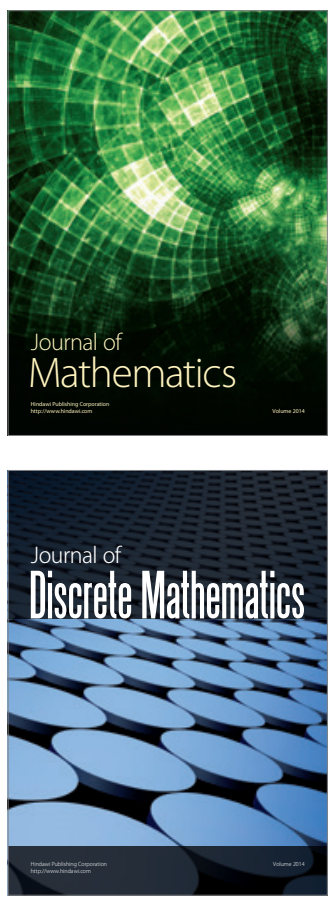

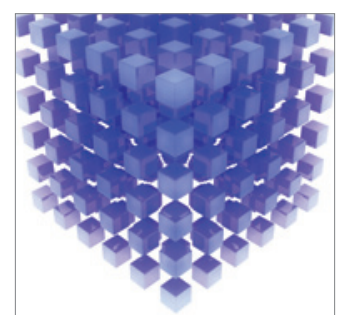

Mathematical Problems in Engineering
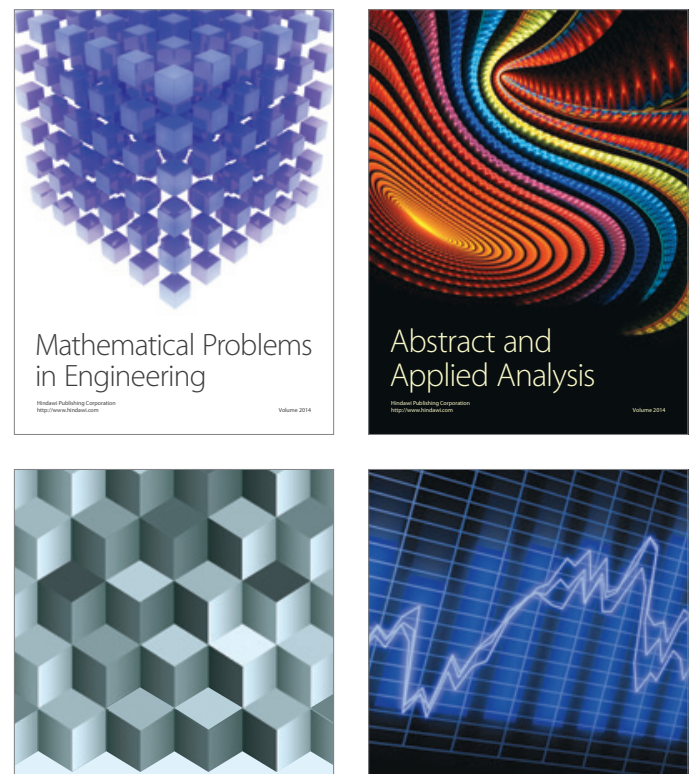

Journal of

Function Spaces

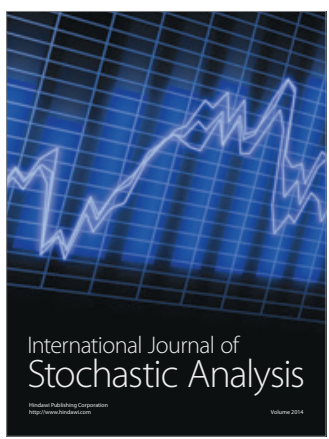

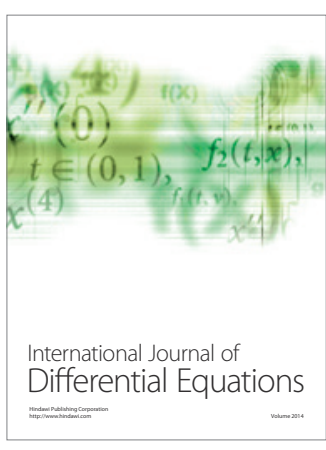
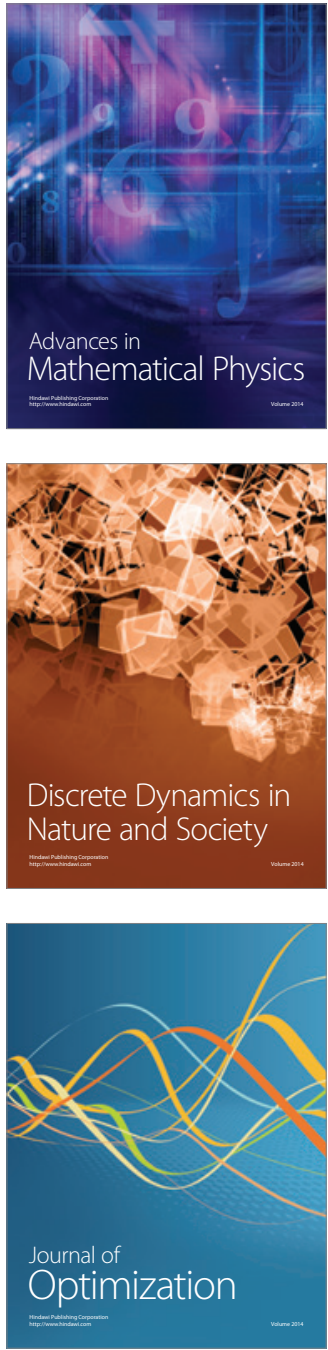